\title{
Tuning Selective Transport of Biomolecules Through Site-Mutated Nucleoporin-Like Protein (NLP) Hydrogels
}

Yun Jung Yang ${ }^{\dagger, \dagger}$, Danielle J. Mai ${ }^{\dagger, \S}$, Shuaili Li ${ }^{\dagger}$, Melody A. Morris ${ }^{\dagger}$, and Bradley D. Olsen ${ }^{*}$,

${ }^{\dagger}$ Department of Chemical Engineering, Massachusetts Institute of Technology, Cambridge, MA

02139, USA

${ }^{\ddagger}$ Department of Biological Engineering, Inha University, 100 Inha-ro, Nam-gu, Incheon, 22212,

Republic of Korea

${ }^{\S}$ Department of Chemical Engineering, Stanford University, Stanford, CA 94305, USA

*Correspondence: Professor Bradley D. Olsen, Department of Chemical Engineering,

Massachusetts Institute of Technology, 77 Massachusetts Ave 66-558A, Cambridge, MA, 02139, USA.

Tel: +1 617-715-4548

E-mail address: bdolsen@mit.edu 


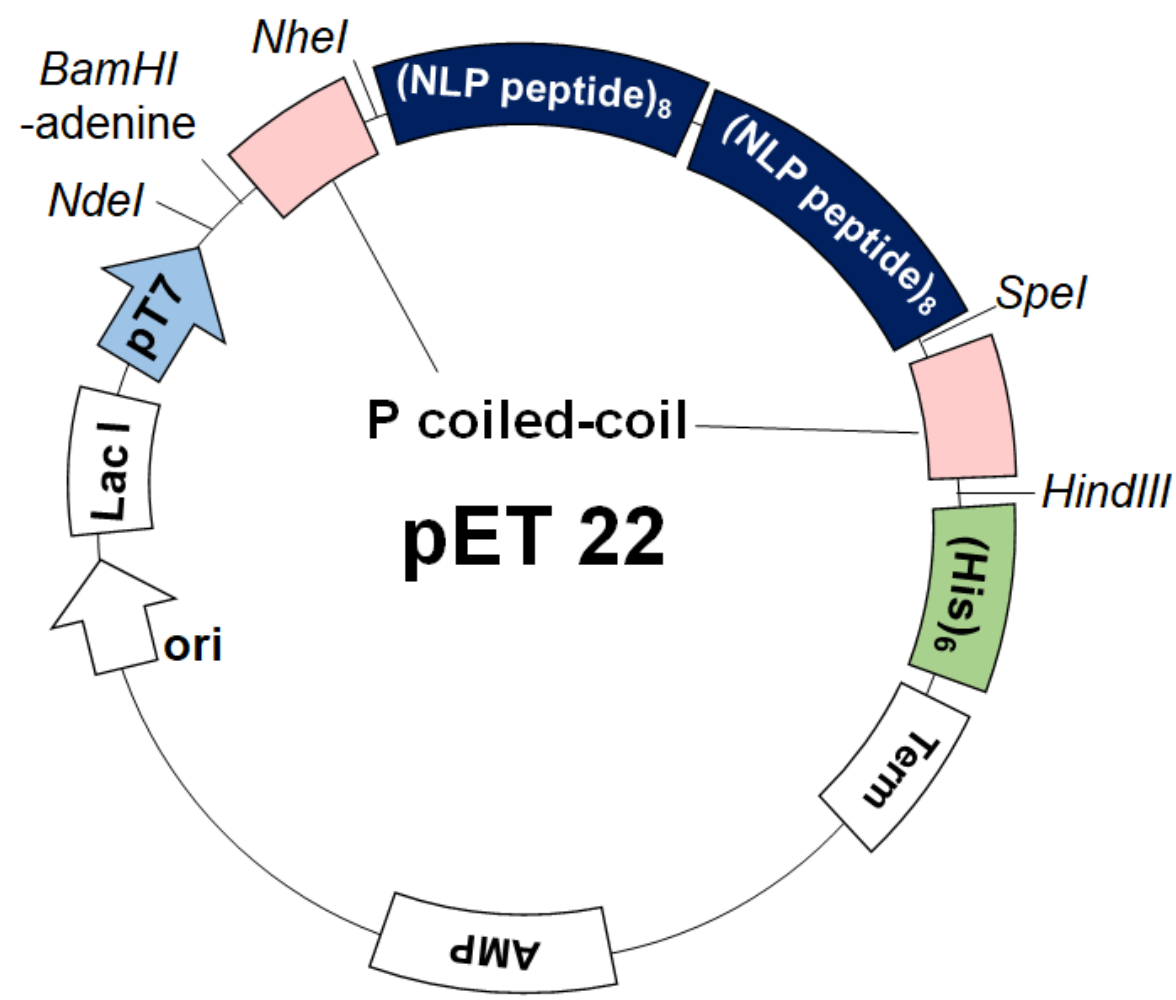

Figure S1. Vector construction for P-NLP-P expression ${ }^{20}$ 
Table S1. Expression yield of P-\#NLP-P proteins. No expression was observed for 3NLP, 12NLP, and 13NLP. 7NLP and 8NLP had very low expression level, which deterred further hydrogel fabrication. The experiment in this paper was conducted with the other NLPs that showed relatively high expression yield.

\begin{tabular}{|c|l|c|}
\hline & \multicolumn{1}{|c|}{ Mid-block Sequence } & Yield (mg/L) \\
\hline P-1NLP-P & PAFSFGAKPDEKKDDDTSK & $50 \pm 15$ \\
\hline P-2NLP-P & PAFSFGAKPDEKKDSDTSK & $25 \pm 9$ \\
\hline P-3NLP-P & PAFSFGASPDEKKDSDTSK & $\begin{array}{c}\text { No detectable } \\
\text { expression }\end{array}$ \\
\hline P-4NLP-P & PAFSFGAEPDEKKDSDTSK & $18 \pm 4$ \\
\hline P-5NLP-P & PAFSFGAKPDEKKDSDTSS & $30 \pm 9$ \\
\hline P-6NLP-P & PAFSFGAKPDEKKDSDTSE & $50 \pm 10$ \\
\hline P-7NLP-P & PAYSFGAKPDEKKDSDTSK & $3 \pm 2$ \\
\hline P-8NLP-P & PAYSYGAKPDEKKDSDTSK & $3 \pm 2$ \\
\hline P-9NLP-P & SAFSFGAKPDEKKDSDTSK & $50 \pm 5$ \\
\hline P-10NLP-P & PSFSFGAKPDEKKDSDTSK & $26 \pm 8$ \\
\hline P-11NLP-P & PAFSFGSKPDEKKDSDTSK & $20 \pm 5$ \\
\hline P-12NLP-P & PAFSFGAKPKKKKKSKTSK & $\begin{array}{c}\text { No detectable } \\
\text { expression }\end{array}$ \\
\hline P-13NLP-P & PAFSFGAKPDEDEDSDTSK & $\begin{array}{c}\text { No detectable } \\
\text { expression }\end{array}$ \\
\hline
\end{tabular}



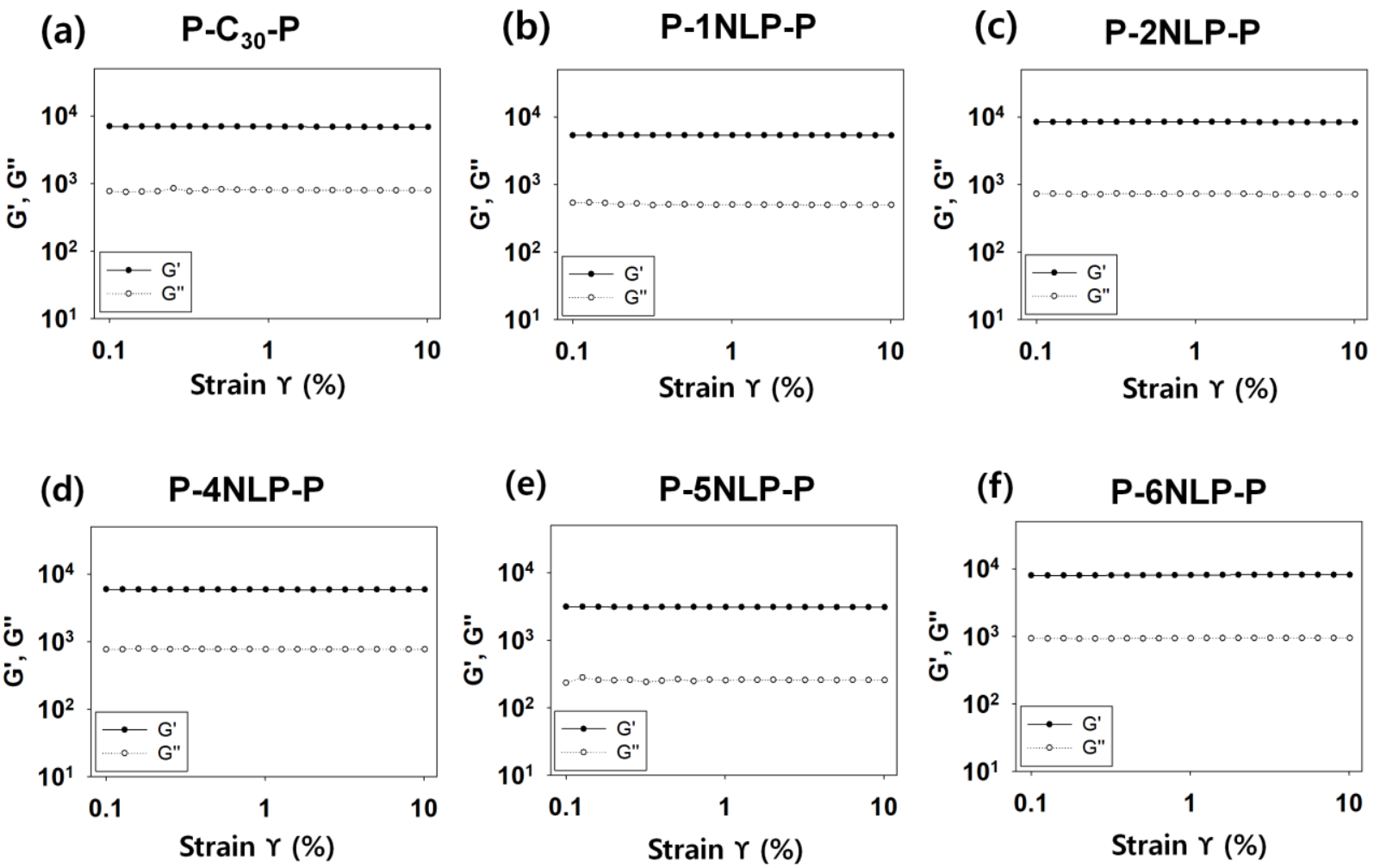

(g) P-9NLP-P
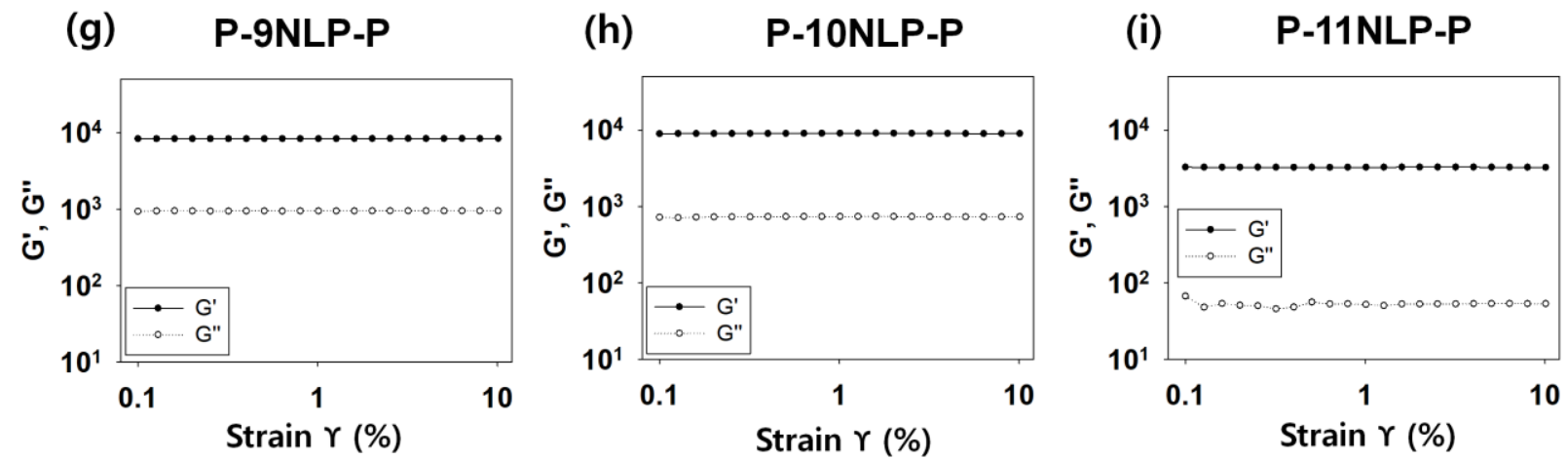

Figure S2. Strain-sweep rheology data for $\mathrm{P}-\mathrm{C}_{30}-\mathrm{P}$ and NLP hydrogels 

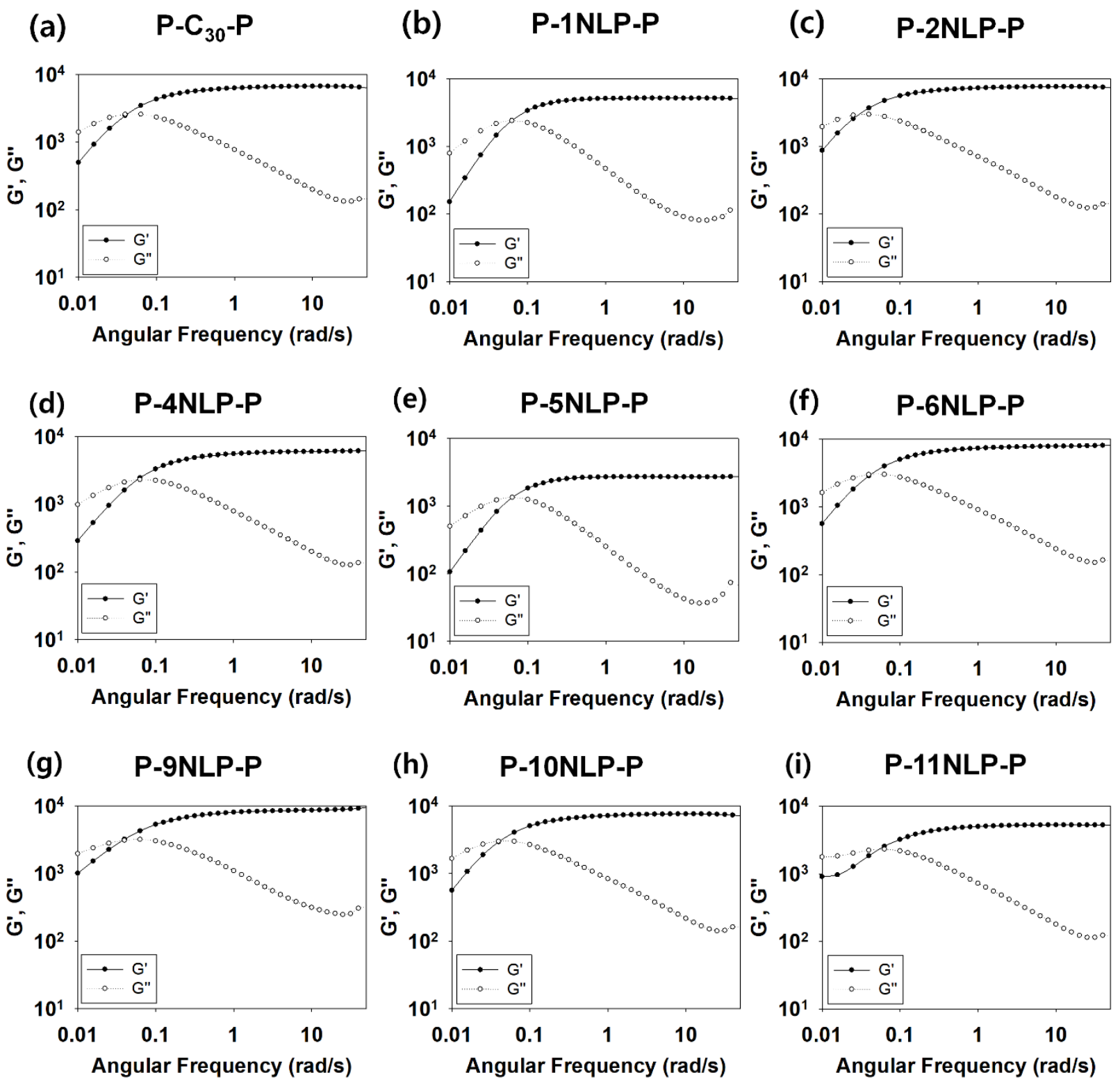

Figure S3. Frequency sweep of $\mathrm{P}-\mathrm{C}_{30}-\mathrm{P}$ and NLP hydrogels at linear oscillatory shear rheology region. 
Table S2. Supporting table for Figure 6 in the main text. The values are the maximum intensity of NTF2-GFP and BSA near gel interface $(0-500 \mu \mathrm{m}$ depth) and the mean integration within the gel of NTF2-GFP and BSA at $60 \mathrm{~min}$. The values were averaged from three different 1D transport assay batches, and the error bar shows standard error.

\begin{tabular}{|c|c|c|c|c|}
\hline & \multicolumn{2}{|c|}{ NTF2-GFP } & \multicolumn{2}{c|}{ BSA } \\
\hline & $\begin{array}{c}\text { Mean maximum } \\
\text { intensity }( \pm \\
\text { standard error })\end{array}$ & $\begin{array}{c}\text { Mean } \\
\text { integration }( \pm \\
\text { standard error) }\end{array}$ & $\begin{array}{c}\text { Mean maximum } \\
\text { intensity }( \pm \\
\text { standard error })\end{array}$ & $\begin{array}{c}\text { Mean } \\
\text { integration }( \pm \\
\text { standard error })\end{array}$ \\
\hline P-1NLP-P & $1.03 \pm 0.01$ & $407 \pm 15$ & $1.02 \pm 0.03$ & $439 \pm 34$ \\
\hline P-2NLP-P & $2.89 \pm 0.05$ & $1062 \pm 81$ & $1.05 \pm 0.02$ & $578 \pm 62$ \\
\hline P-4NLP-P & $1.05 \pm 0.02$ & $366 \pm 44$ & $1.03 \pm 0.04$ & $452 \pm 9$ \\
\hline P-5NLP-P & $1.04 \pm 0.01$ & $434 \pm 89$ & $0.99 \pm 0.06$ & $482 \pm 101$ \\
\hline P-6NLP-P & $2.02 \pm 0.11$ & $986 \pm 20$ & $1.04 \pm 0.06$ & $644 \pm 47$ \\
\hline P-9NLP-P & $1.97 \pm 0.24$ & $832 \pm 135$ & $1.09 \pm 0.01$ & $562 \pm 62$ \\
\hline P-10NLP-P & $3.36 \pm 0.32$ & $1066 \pm 96$ & $1.02 \pm 0.01$ & $529 \pm 8$ \\
\hline P-11NLP-P & $1.07 \pm 0.02$ & $441 \pm 8$ & $1.13 \pm 0.07$ & $564 \pm 48$ \\
\hline P-C30-P & $1.05 \pm 0.02$ & $614 \pm 81$ & $0.96 \pm 0.06$ & $541 \pm 52$ \\
\hline
\end{tabular}



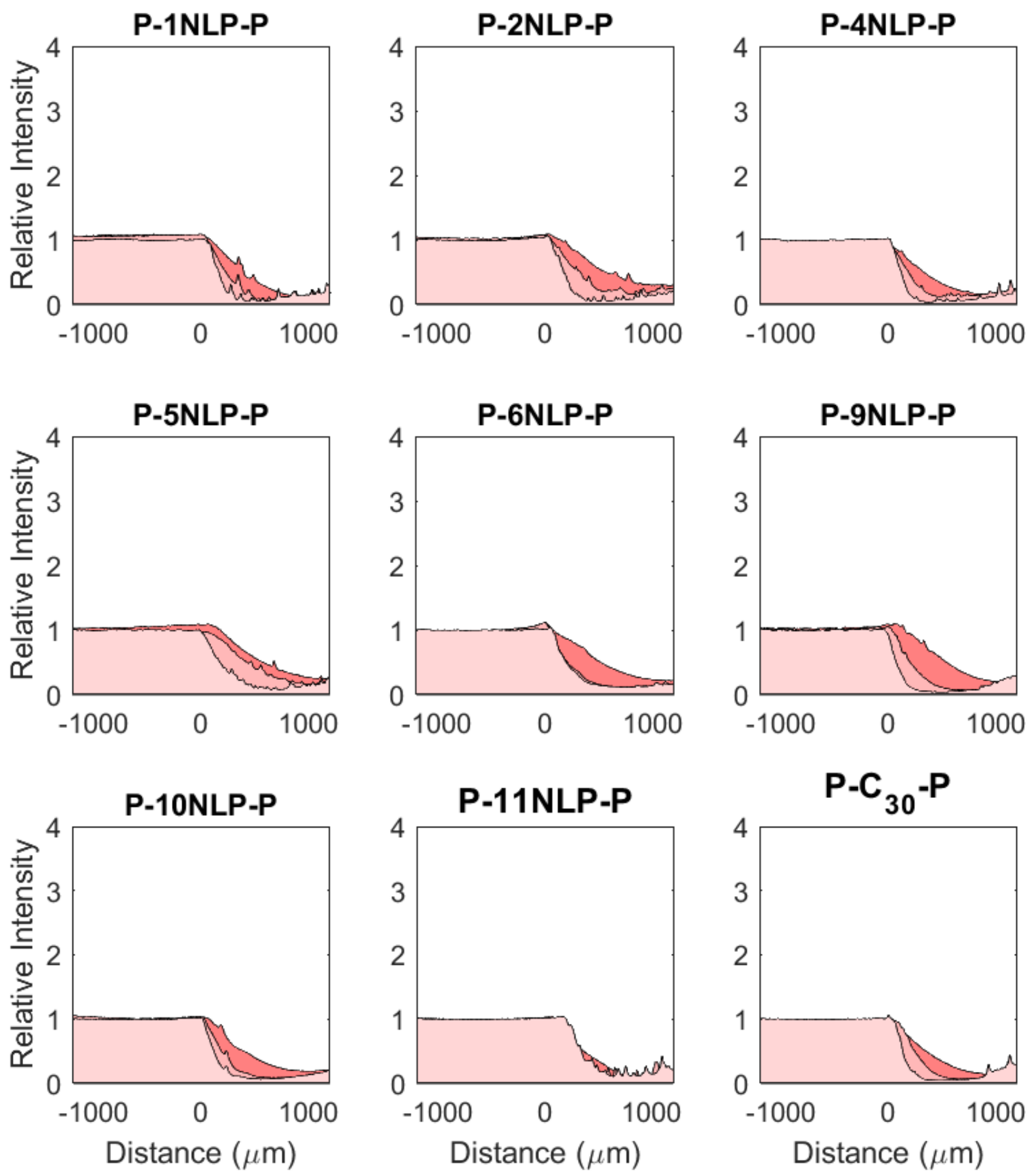

Figure S4. Fluorescence intensity measurements of BSA across the P-xNLP-P and P-C $\mathrm{C}_{30}-\mathrm{P}$ hydrogels. ' 0 ' in distance indicates the hydrogel interface. The plots are derived from the time lapse image measurements in Figure 4a and 5a, derived from the 1D transport assay. 

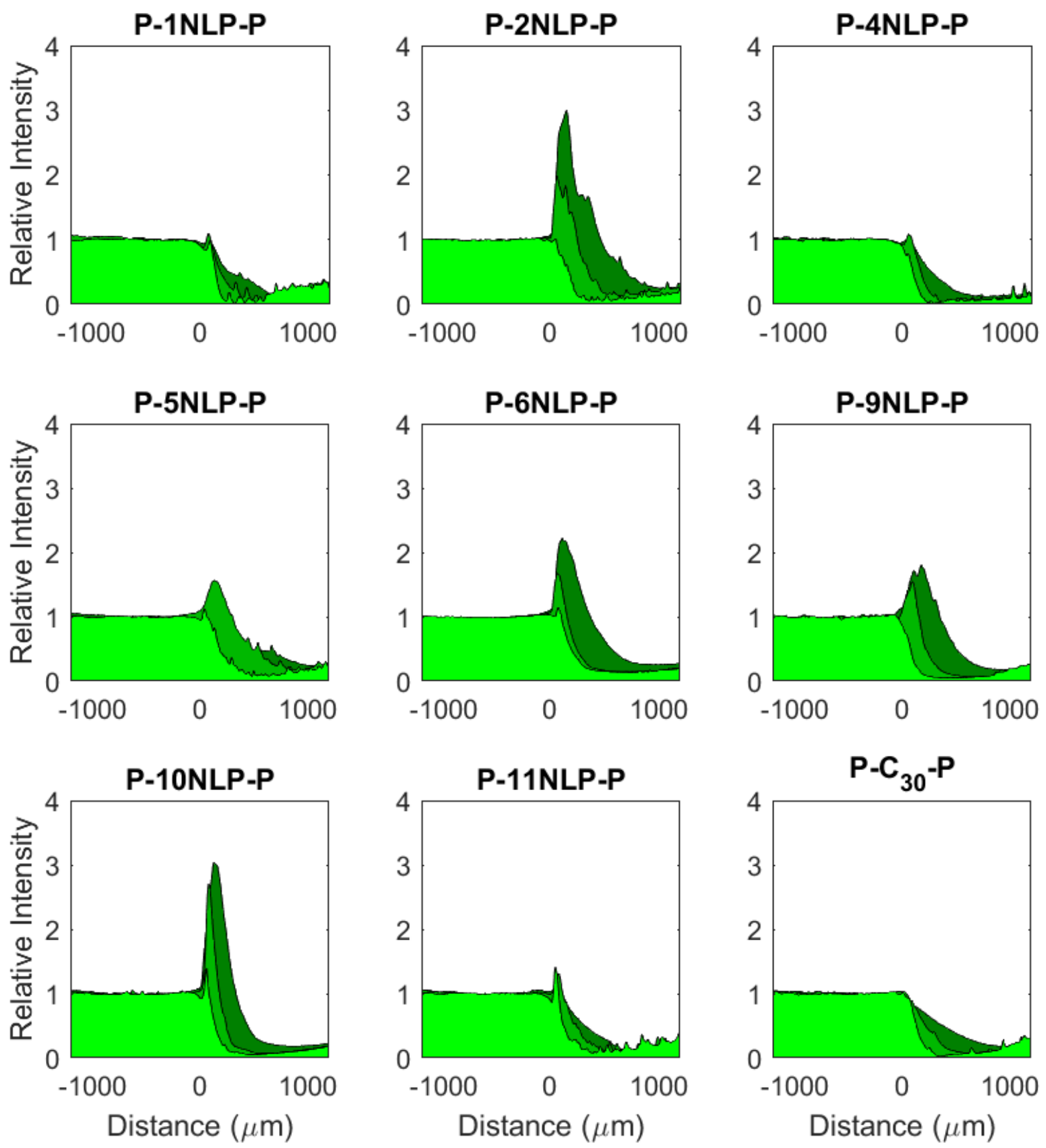

Figure S5. Selective permeability test of NTF2-GFP performed across the NLP and $\mathrm{P}_{-} \mathrm{C}_{30}-\mathrm{P}$ hydrogels. ' 0 ' in distance indicates the hydrogel interface. The plots are derived from the time lapse image measurements in Figure 4b and 5b, derived from the 1D transport assay. 


\section{Recombinant protein sequence information in this paper}

\section{P-1NLP-P}

MDIGINSDPAPQMLRELQETNAALQDVRELLRQQVKEITFLKNTVMESDASGASPAFS FGAKPDEKKDDDTSKPAFSFGAKPDEKKDDDTSKPAFSFGAKPDEKKDDDTSKPAFSF GAKPDEKKDDDTSKPAFSFGAKPDEKKDDDTSKPAFSFGAKPDEKKDDDTSKPAFSFG AKPDEKKDDDTSKPAFSFGAKPDEKKDDDTSKTSPAFSFGAKPDEKKDDDTSKPAFSF GAKPDEKKDDDTSKPAFSFGAKPDEKKDDDTSKPAFSFGAKPDEKKDDDTSKPAFSFG AKPDEKKDDDTSKPAFSFGAKPDEKKDDDTSKPAFSFGAKPDEKKDDDTSKPAFSFGA KPDEKKDDDTSKTSAPQMLRELQETNAALQDVRELLRQQVKEITFLKNTVMESDASG GKLAAALEHHHHHH

\section{P-2NLP-P}

MDIGINSDPAPQMLRELQETNAALQDVRELLRQQVKEITFLKNTVMESDASGASPAFS FGAKPDEKKDSDTSKPAFSFGAKPDEKKDSDTSKPAFSFGAKPDEKKDSDTSKPAFSFG AKPDEKKDSDTSKPAFSFGAKPDEKKDSDTSKPAFSFGAKPDEKKDSDTSKPAFSFGA KPDEKKDSDTSKPAFSFGAKPDEKKDSDTSKTSPAFSFGAKPDEKKDSDTSKPAFSFGA KPDEKKDSDTSKPAFSFGAKPDEKKDSDTSKPAFSFGAKPDEKKDSDTSKPAFSFGAKP DEKKDSDTSKPAFSFGAKPDEKKDSDTSKPAFSFGAKPDEKKDSDTSKPAFSFGAKPDE KKDSDTSKTSAPQMLRELQETNAALQDVRELLRQQVKEITFLKNTVMESDASGGKLA AALEHHHHHH

\section{P-3NLP-P}

MDIGINSDPAPQMLRELQETNAALQDVRELLRQQVKEITFLKNTVMESDASGASPAFS FGASPDEKKDSDTSKPAFSFGASPDEKKDSDTSKPAFSFGASPDEKKDSDTSKPAFSFG ASPDEKKDSDTSKPAFSFGASPDEKKDSDTSKPAFSFGASPDEKKDSDTSKPAFSFGASP DEKKDSDTSKPAFSFGASPDEKKDSDTSKTSPAFSFGASPDEKKDSDTSKPAFSFGASP DEKKDSDTSKPAFSFGASPDEKKDSDTSKPAFSFGASPDEKKDSDTSKPAFSFGASPDE KKDSDTSKPAFSFGASPDEKKDSDTSKPAFSFGASPDEKKDSDTSKPAFSFGASPDEKK DSDTSKTSAPQMLRELQETNAALQDVRELLRQQVKEITFLKNTVMESDASGGKLAAA LEHHHHHH

\section{P-4NLP-P}

MDIGINSDPAPQMLRELQETNAALQDVRELLRQQVKEITFLKNTVMESDASGASPAFS FGAEPDEKKDSDTSKPAFSFGAEPDEKKDSDTSKPAFSFGAEPDEKKDSDTSKPAFSFG AEPDEKKDSDTSKPAFSFGAEPDEKKDSDTSKPAFSFGAEPDEKKDSDTSKPAFSFGAE PDEKKDSDTSKPAFSFGAEPDEKKDSDTSKTSPAFSFGAEPDEKKDSDTSKPAFSFGAE PDEKKDSDTSKPAFSFGAEPDEKKDSDTSKPAFSFGAEPDEKKDSDTSKPAFSFGAEPD EKKDSDTSKPAFSFGAEPDEKKDSDTSKPAFSFGAEPDEKKDSDTSKPAFSFGAEPDEK KDSDTSKTSAPQMLRELQETNAALQDVRELLRQQVKEITFLKNTVMESDASGGKLAA ALEHHHHHH

P-5NLP-P 
MDIGINSDPAPQMLRELQETNAALQDVRELLRQQVKEITFLKNTVMESDASGASPAFS FGAKPDEKKDSDTSSPAFSFGAKPDEKKDSDTSSPAFSFGAKPDEKKDSDTSSPAFSFG AKPDEKKDSDTSSPAFSFGAKPDEKKDSDTSSPAFSFGAKPDEKKDSDTSSPAFSFGAK PDEKKDSDTSSPAFSFGAKPDEKKDSDTSSTSPAFSFGAKPDEKKDSDTSSPAFSFGAKP DEKKDSDTSSPAFSFGAKPDEKKDSDTSSPAFSFGAKPDEKKDSDTSSPAFSFGAKPDE KKDSDTSSPAFSFGAKPDEKKDSDTSSPAFSFGAKPDEKKDSDTSSPAFSFGAKPDEKK DSDTSSTSAPQMLRELQETNAALQDVRELLRQQVKEITFLKNTVMESDASGGKLAAA LEHHHHHH

\section{P-6NLP-P}

MDIGINSDPAPQMLRELQETNAALQDVRELLRQQVKEITFLKNTVMESDASGASPAFS FGAKPDEKKDSDTSEPAFSFGAKPDEKKDSDTSEPAFSFGAKPDEKKDSDTSEPAFSFG AKPDEKKDSDTSEPAFSFGAKPDEKKDSDTSEPAFSFGAKPDEKKDSDTSEPAFSFGAK PDEKKDSDTSEPAFSFGAKPDEKKDSDTSETSPAFSFGAKPDEKKDSDTSEPAFSFGAK PDEKKDSDTSEPAFSFGAKPDEKKDSDTSEPAFSFGAKPDEKKDSDTSEPAFSFGAKPD EKKDSDTSEPAFSFGAKPDEKKDSDTSEPAFSFGAKPDEKKDSDTSEPAFSFGAKPDEK KDSDTSETSAPQMLRELQETNAALQDVRELLRQQVKEITFLKNTVMESDASGGKLAA ALEHHHHHH

\section{P-7NLP-P}

MDIGINSDPAPQMLRELQETNAALQDVRELLRQQVKEITFLKNTVMESDASGASPAYS FGAKPDEKKDSDTSKPAYSFGAKPDEKKDSDTSKPAYSFGAKPDEKKDSDTSKPAYSF GAKPDEKKDSDTSKPAYSFGAKPDEKKDSDTSKPAYSFGAKPDEKKDSDTSKPAYSFG AKPDEKKDSDTSKPAYSFGAKPDEKKDSDTSKTSPAYSFGAKPDEKKDSDTSKPAYSF GAKPDEKKDSDTSKPAYSFGAKPDEKKDSDTSKPAYSFGAKPDEKKDSDTSKPAYSFG AKPDEKKDSDTSKPAYSFGAKPDEKKDSDTSKPAYSFGAKPDEKKDSDTSKPAYSFGA KPDEKKDSDTSKTSAPQMLRELQETNAALQDVRELLRQQVKEITFLKNTVMESDASG GKLAAALEHHHHHH

\section{P-8NLP-P}

MDIGINSDPAPQMLRELQETNAALQDVRELLRQQVKEITFLKNTVMESDASGASPAYS YGAKPDEKKDSDTSKPAYSYGAKPDEKKDSDTSKPAYSYGAKPDEKKDSDTSKPAYS YGAKPDEKKDSDTSKPAYSYGAKPDEKKDSDTSKPAYSYGAKPDEKKDSDTSKPAYS YGAKPDEKKDSDTSKPAYSYGAKPDEKKDSDTSKTSPAYSYGAKPDEKKDSDTSKPAY SYGAKPDEKKDSDTSKPAYSYGAKPDEKKDSDTSKPAYSYGAKPDEKKDSDTSKPAYS YGAKPDEKKDSDTSKPAYSYGAKPDEKKDSDTSKPAYSYGAKPDEKKDSDTSKPAYS YGAKPDEKKDSDTSKTSAPQMLRELQETNAALQDVRELLRQQVKEITFLKNTVMESD ASGGKLAAALEHHHHHH

\section{P-9NLP-P}

MDIGINSDPAPQMLRELQETNAALQDVRELLRQQVKEITFLKNTVMESDASGASSAFS FGAKPDEKKDSDTSKSAFSFGAKPDEKKDSDTSKSAFSFGAKPDEKKDSDTSKSAFSF GAKPDEKKDSDTSKSAFSFGAKPDEKKDSDTSKSAFSFGAKPDEKKDSDTSKSAFSFG 
AKPDEKKDSDTSKSAFSFGAKPDEKKDSDTSKTSSAFSFGAKPDEKKDSDTSKSAFSF GAKPDEKKDSDTSKSAFSFGAKPDEKKDSDTSKSAFSFGAKPDEKKDSDTSKSAFSFG AKPDEKKDSDTSKSAFSFGAKPDEKKDSDTSKSAFSFGAKPDEKKDSDTSKSAFSFGA KPDEKKDSDTSKTSAPQMLRELQETNAALQDVRELLRQQVKEITFLKNTVMESDASG GKLAAALEHHHHHH

\section{P-10NLP-P}

MDIGINSDPAPQMLRELQETNAALQDVRELLRQQVKEITFLKNTVMESDASGASPSFS FGAKPDEKKDSDTSKPSFSFGAKPDEKKDSDTSKPSFSFGAKPDEKKDSDTSKPSFSFG AKPDEKKDSDTSKPSFSFGAKPDEKKDSDTSKPSFSFGAKPDEKKDSDTSKPSFSFGAK PDEKKDSDTSKPSFSFGAKPDEKKDSDTSKTSPSFSFGAKPDEKKDSDTSKPSFSFGAK PDEKKDSDTSKPSFSFGAKPDEKKDSDTSKPSFSFGAKPDEKKDSDTSKPSFSFGAKPD EKKDSDTSKPSFSFGAKPDEKKDSDTSKPSFSFGAKPDEKKDSDTSKPSFSFGAKPDEK KDSDTSKTSAPQMLRELQETNAALQDVRELLRQQVKEITFLKNTVMESDASGGKLAA ALEHHHHHH

\section{P-11NLP-P}

MDIGINSDPAPQMLRELQETNAALQDVRELLRQQVKEITFLKNTVMESDASGASPAFS FGSKPDEKKDSDTSKPAFSFGSKPDEKKDSDTSKPAFSFGSKPDEKKDSDTSKPAFSFGS KPDEKKDSDTSKPAFSFGSKPDEKKDSDTSKPAFSFGSKPDEKKDSDTSKPAFSFGSKP DEKKDSDTSKPAFSFGSKPDEKKDSDTSKTSPAFSFGSKPDEKKDSDTSKPAFSFGSKP DEKKDSDTSKPAFSFGSKPDEKKDSDTSKPAFSFGSKPDEKKDSDTSKPAFSFGSKPDE KKDSDTSKPAFSFGSKPDEKKDSDTSKPAFSFGSKPDEKKDSDTSKPAFSFGSKPDEKK DSDTSKTSAPQMLRELQETNAALQDVRELLRQQVKEITFLKNTVMESDASGGKLAAA LEHHHHHH

\section{P-12NLP-P}

MDIGINSDPAPQMLRELQETNAALQDVRELLRQQVKEITFLKNTVMESDASGASPAFS FGAKPKKKKKSKTSKPAFSFGAKPKKKKKSKTSKPAFSFGAKPKKKKKSKTSKPAFSF GAKPKKKKKSKTSKPAFSFGAKPKKKKKSKTSKPAFSFGAKPKKKKKSKTSKPAFSFG AKPKKKKKSKTSKPAFSFGAKPKKKKKSKTSKTSPAFSFGAKPKKKKKSKTSKPAFSF GAKPKKKKKSKTSKPAFSFGAKPKKKKKSKTSKPAFSFGAKPKKKKKSKTSKPAFSFG AKPKKKKKSKTSKPAFSFGAKPKKKKKSKTSKPAFSFGAKPKKKKKSKTSKPAFSFGA KPKKKKKSKTSKTSAPQMLRELQETNAALQDVRELLRQQVKEITFLKNTVMESDASG GKLAAALEHHHHHH

\section{P-13NLP-P}

MDIGINSDPAPQMLRELQETNAALQDVRELLRQQVKEITFLKNTVMESDASGASPAFS FGAKPDEDEDSDTSKPAFSFGAKPDEDEDSDTSKPAFSFGAKPDEDEDSDTSKPAFSFG AKPDEDEDSDTSKPAFSFGAKPDEDEDSDTSKPAFSFGAKPDEDEDSDTSKPAFSFGAK PDEDEDSDTSKPAFSFGAKPDEDEDSDTSKTSPAFSFGAKPDEDEDSDTSKPAFSFGAK PDEDEDSDTSKPAFSFGAKPDEDEDSDTSKPAFSFGAKPDEDEDSDTSKPAFSFGAKPD 
EDEDSDTSKPAFSFGAKPDEDEDSDTSKPAFSFGAKPDEDEDSDTSKPAFSFGAKPDED EDSDTSKTSAPQMLRELQETNAALQDVRELLRQQVKEITFLKNTVMESDASGGKLAA ALEHHHHHH

\section{$\mathbf{P}-\mathbf{C}_{30}-\mathbf{P}$}

MRGSHHHHHHGSGDLAPQMLRELQETNAALQDVRELLRQQVKEITFLKNTVMESDA SGTSYRDPMGAGAGAGPEGAGAGAGPEGAGAGAGPEGAGAGAGPEGAGAGAGPEG AGAGAGPEGAGAGAGPEGAGAGAGPEGAGAGAGPEGAGAGAGPEGARMPTSYRDP MGAGAGAGPEGAGAGAGPEGAGAGAGPEGAGAGAGPEGAGAGAGPEGAGAGAGP EGAGAGAGPEGAGAGAGPEGAGAGAGPEGAGAGAGPEGARMPTSYRDPMGAGAG AGPEGAGAGAGPEGAGAGAGPEGAGAGAGPEGAGAGAGPEGAGAGAGPEGAGAGA GPEGAGAGAGPEGAGAGAGPEGAGAGAGPEGARMPTSGSGDLAPQMLRELQETNA ALQDVRELLRQQVKEITFLKNTVMESDASGKLN

\section{NTF2-GFP}

MGDKPIWEQIGSSFIQHYYQLFDNDRTQLGAIYIDASCLTWEGQQFQGKAAIVEKLSSL PFQKIQHSITAQDHQPTPDSCIISMVVGQLKADEDPIMGFHQMFLLKNINDAWVCTND MFRLALHNFGTSGSACELMVSKGEELFTGVVPILVELDGDVNGHKFSVRGEGEGDAT NGKLTLKFICTTGKLPVPWPTLVTTLTYGVQCFSRYPDHMKQHDFFKSAMPEGYVQE RTISFKDDGTYKTRAEVKFEGDTLVNRIELKGIDFKEDGNILGHKLEYNFNSHNVYITA DKQKNGIKANFKIRHNVEDGSVQLADHYQQNTPIGDGPVLLPDNHYLSTQSALSKDP NEKRDHMVLLEFVTAAGITHGMDELYKGSRSHHHHHH

\section{Matlab Code for Data Processing:}

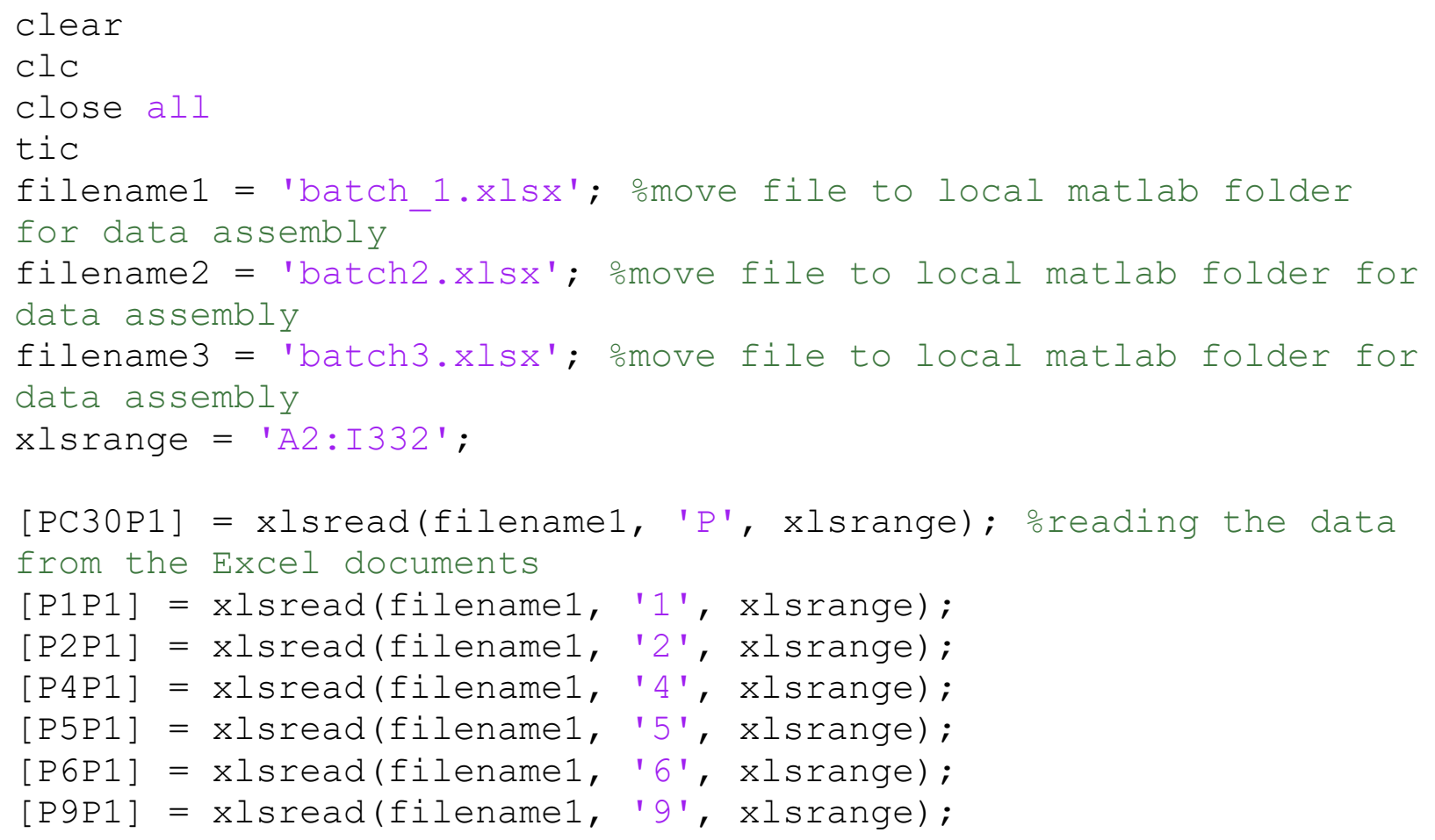




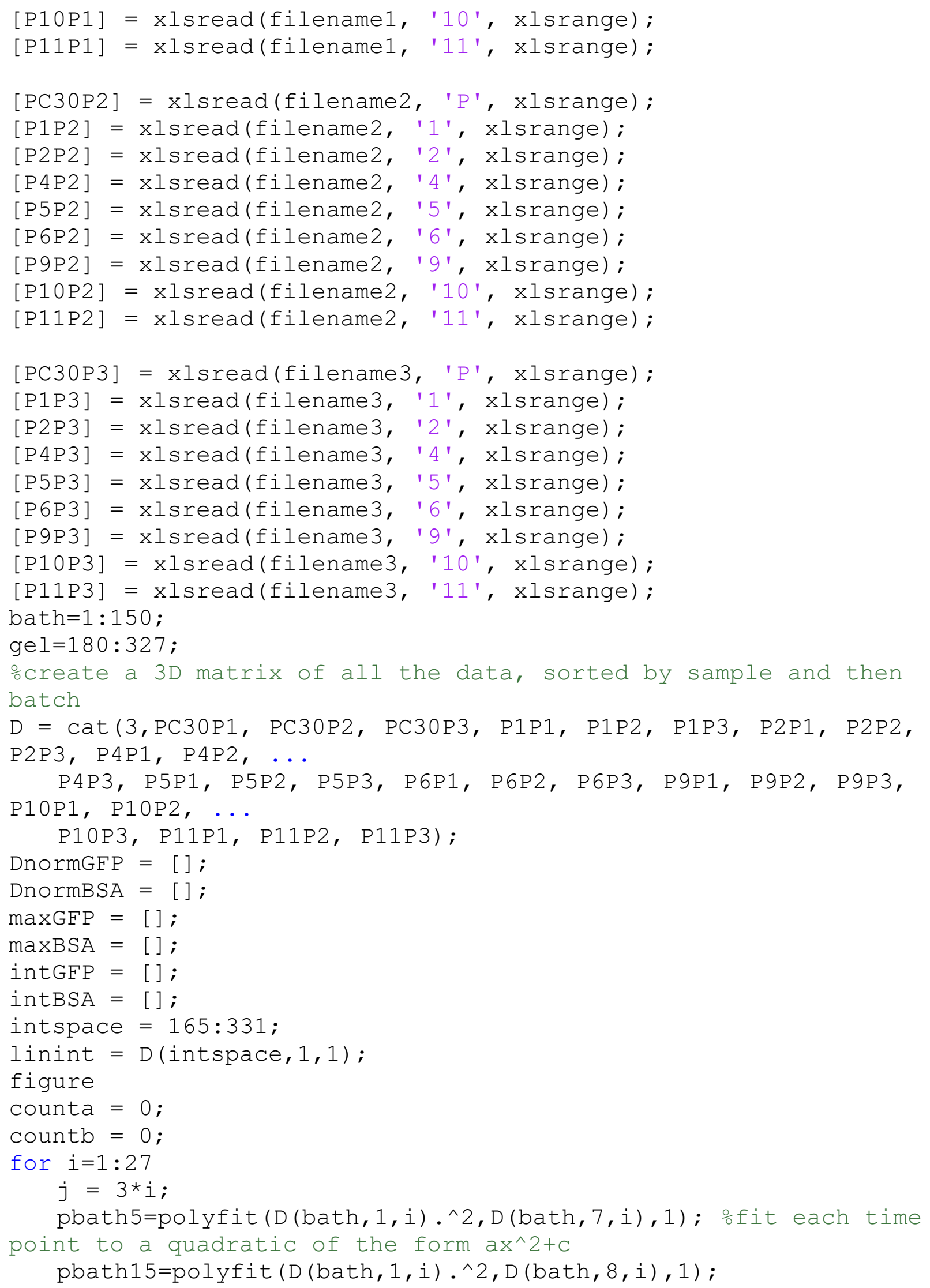


pbath $60=$ polyfit $(\mathrm{D}($ bath $, 1, i) . \wedge 2, D($ bath, 9, i $), 1)$;

pgell=[min $(\mathrm{D}(\mathrm{gel}, 7, \mathrm{i})) \min (\mathrm{D}(\mathrm{gel}, 8, i)) \min (\mathrm{D}(\mathrm{gel}, 9, \mathrm{i}))]$;

pgel=min (pgell);

$\mathrm{x} 1=\mathrm{D}(:, 1, \mathrm{i})$;

$\mathrm{f} 1=$ polyval (pbath5, x1.^2);

pbathBSA5 = polyfit $\left(\mathrm{D}(\right.$ bath $, 1, i) \cdot{ }^{\wedge} 2, \mathrm{D}($ bath $\left., 2, i), 1\right)$;

pbathBSA15 = polyfit $\left(\mathrm{D}(\right.$ bath $, 1, i) \cdot{ }^{\wedge} 2, \mathrm{D}($ bath $\left., 3, i), 1\right)$;

pbathBSA60 = polyfit $\left(\mathrm{D}(\right.$ bath, $1, i) .^{\wedge} 2, \mathrm{D}($ bath $\left., 4, i), 1\right)$;

$\mathrm{f} 2$ = polyval (pbathBSA5, $\left.\mathrm{x} 1 .{ }^{\wedge} 2\right)$;

pgelBSA $1=[\min (D(g e l, 2, i)), \min (D(g e l, 3, i)), \min (D(g e l, 4, i))]$; pgelBSA=min (pgelBSA 1$)$;

DnormGFP $=$ [DnormGFP, $(\mathrm{D}(:, 7, \mathrm{i})-(\mathrm{f} 1-$

pbath5 (2) +pgel1(1))) / (pbath5 (2) -pgel1(1)), ...

$(\mathrm{D}($ : , 8, i $)-($ f1-pbath5 (2) +pgel1 (1))) /(pbath15 (2)-pgel1 (1)),

$(D(:, 9, i)-(f 1-p b a t h 5(2)+\operatorname{pgel1}(1))) \ldots$

/ (pbath60(2)-pgel1(1))]; operform normalization for GFP

DnormBSA $=[$ DnormBSA, $(\mathrm{D}(:, 2, \mathrm{i})-(\mathrm{f} 2-$

pbathBSA5 (2) +pgelBSA1 (1)) ) / (pbathBSA5 (2) -pgelBSA1 (1)), ...

$(\mathrm{D}(:, 3, i)-($ f2-pbathBSA5 (2) +pgelBSA1 (1)) ) / (pbathBSA5 (2) -

pgelBSA1 (1)), (D $(:, 4, i)-(f 2-\ldots$

pbathBSA5 (2) +pgelBSA1 (1))) / (pbathBSA5 (2) -

pgelBSA1 (1)) ] ; onormalization for BSA

$\operatorname{maxGFP}=[\operatorname{maxGFP}, \max (\operatorname{DnormGFP}(:, j-2)), \max (\operatorname{DnormGFP}(:, j-1))$, $\max (\operatorname{DnormGFP}(:, j))]$; ocalculate the maximum values of each

normalized curve

$\operatorname{maxBSA}=[\operatorname{maxBSA}, \max (\operatorname{DnormBSA}(:, j-2)), \max (\operatorname{DnormBSA}(:, j-1))$, $\max (\operatorname{DnormBSA}(:, j))]$;

intGFP $=$ [intGFP, trapz(linint, DnormGFP(intspace, j-2)),

trapzllinint, ...

DnormGFP(intspace,j-1)), trapz(linint,

DnormGFP (intspace, j)) ] ;

ocalculate the integration through the gel

intBSA $=$ [intBSA, trapz(linint, DnormBSA (intspace,j-2)), trapzllinint, ...

DnormBSA (intspace,j-1)), trapz(linint,

DnormBSA (intspace, j)) ] ;

end

meanmaxGFP $=[]$;

$\mathrm{SDmaxGFP}=[]$;

meanmaxGFP60 = [];

$\mathrm{SDmaxGFP} 60=[]$;

meanmaxBSA $=[]$;

$\mathrm{SDmaxBSA}=[]$;

meanmaxBSA60 = [];

$\mathrm{SDmaxBSA} 60=[$ ]

meanintGFP $=$ [];

SDintGFP $=[]$; 


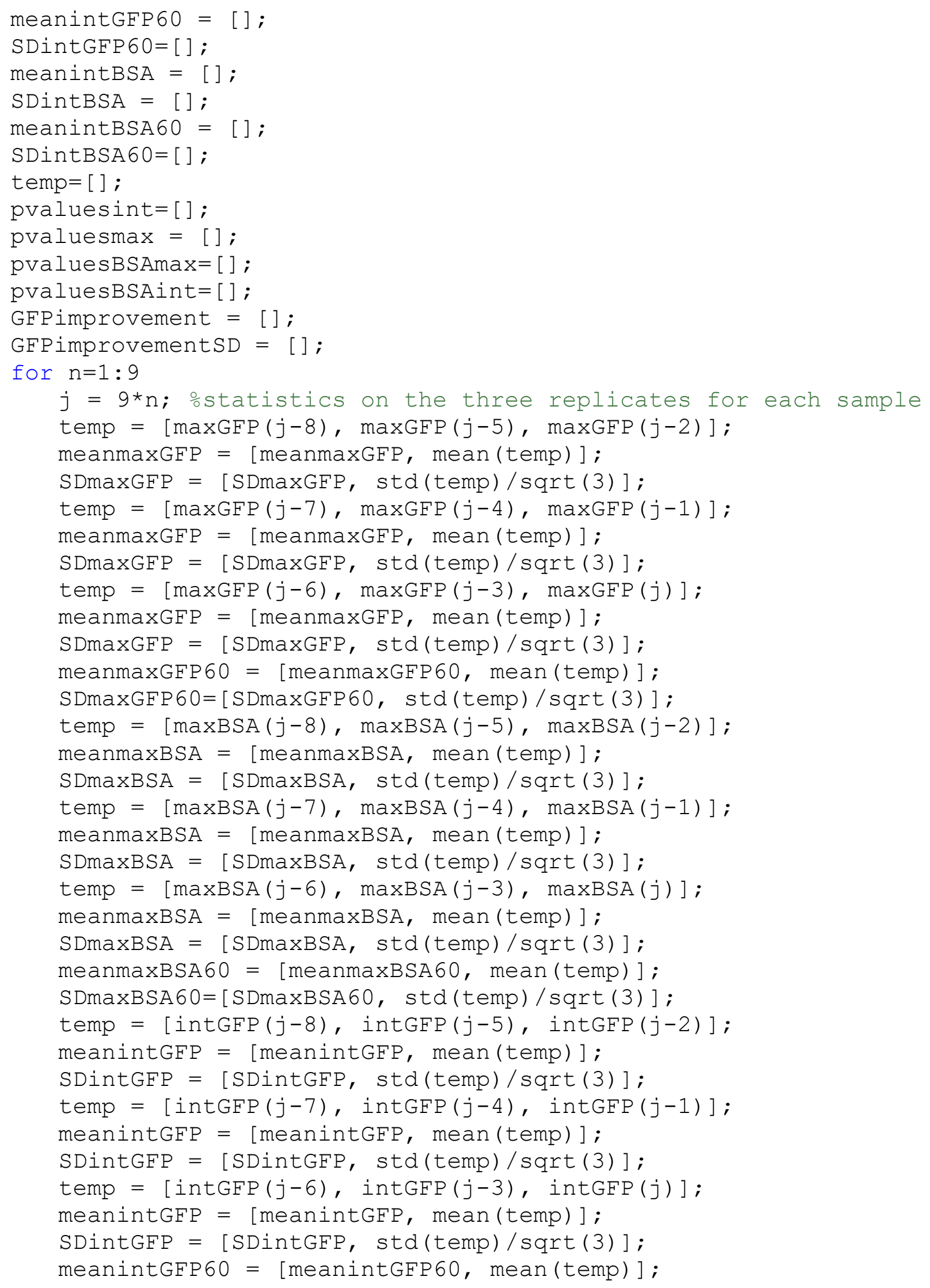




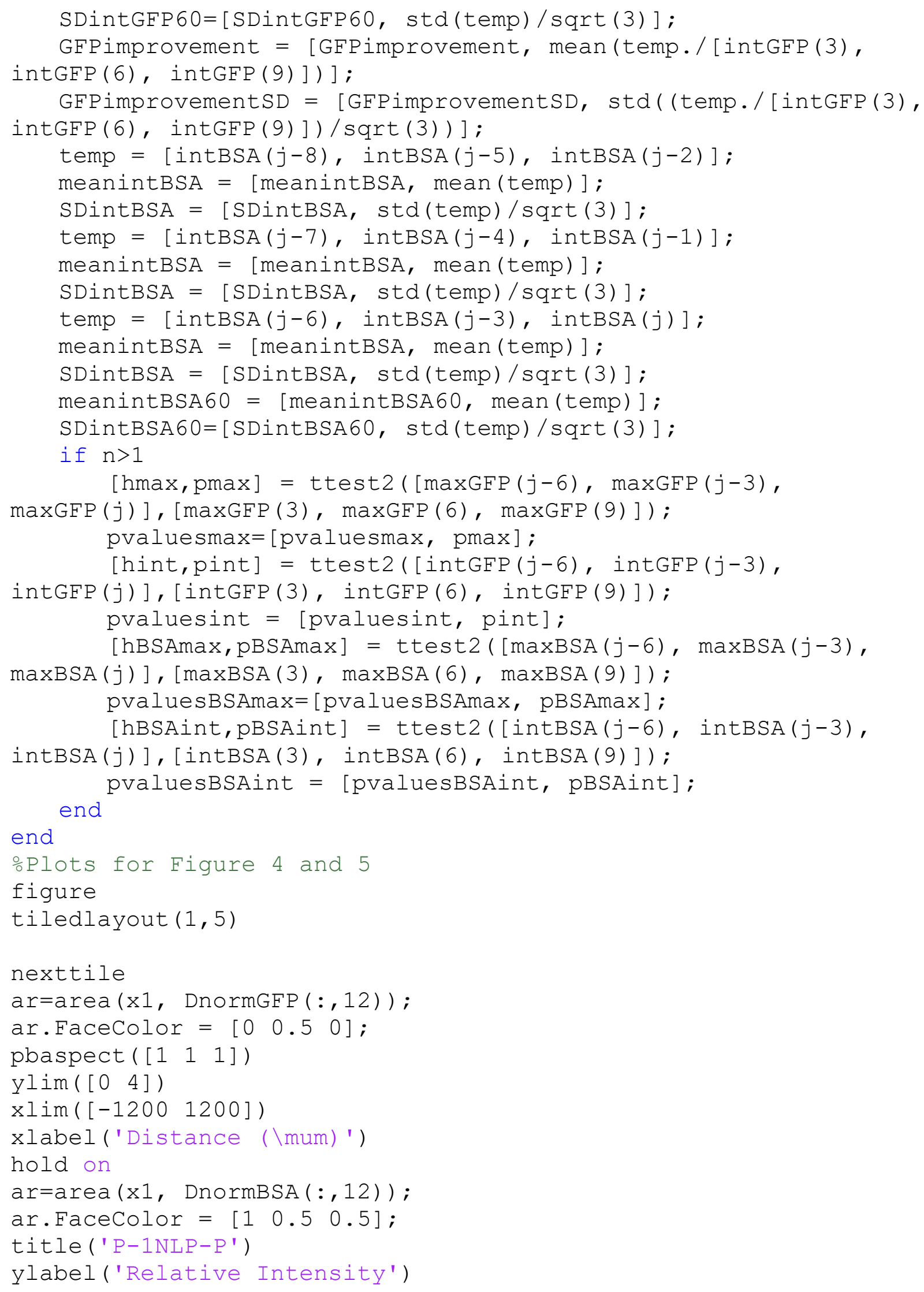




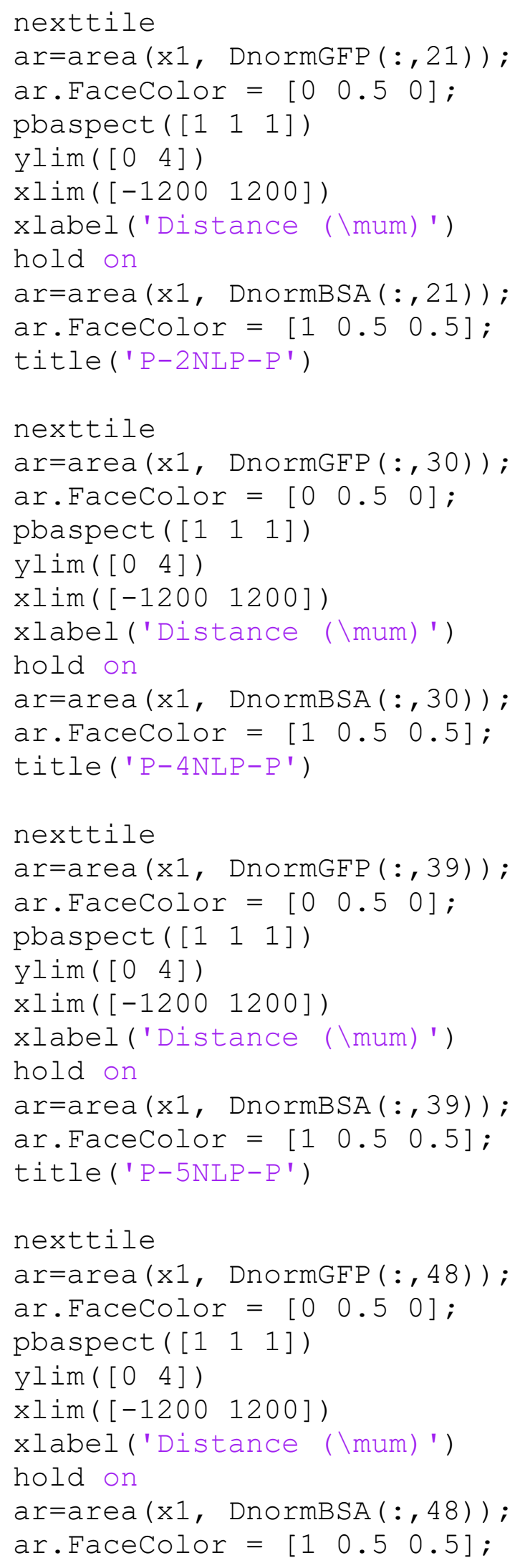




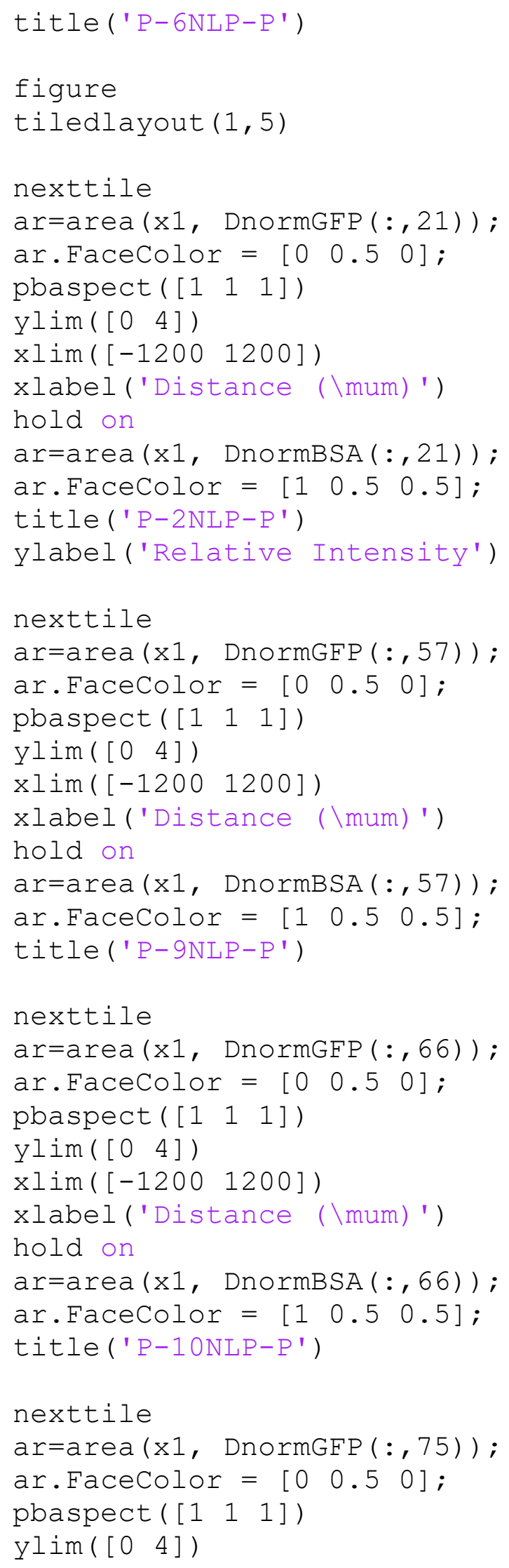




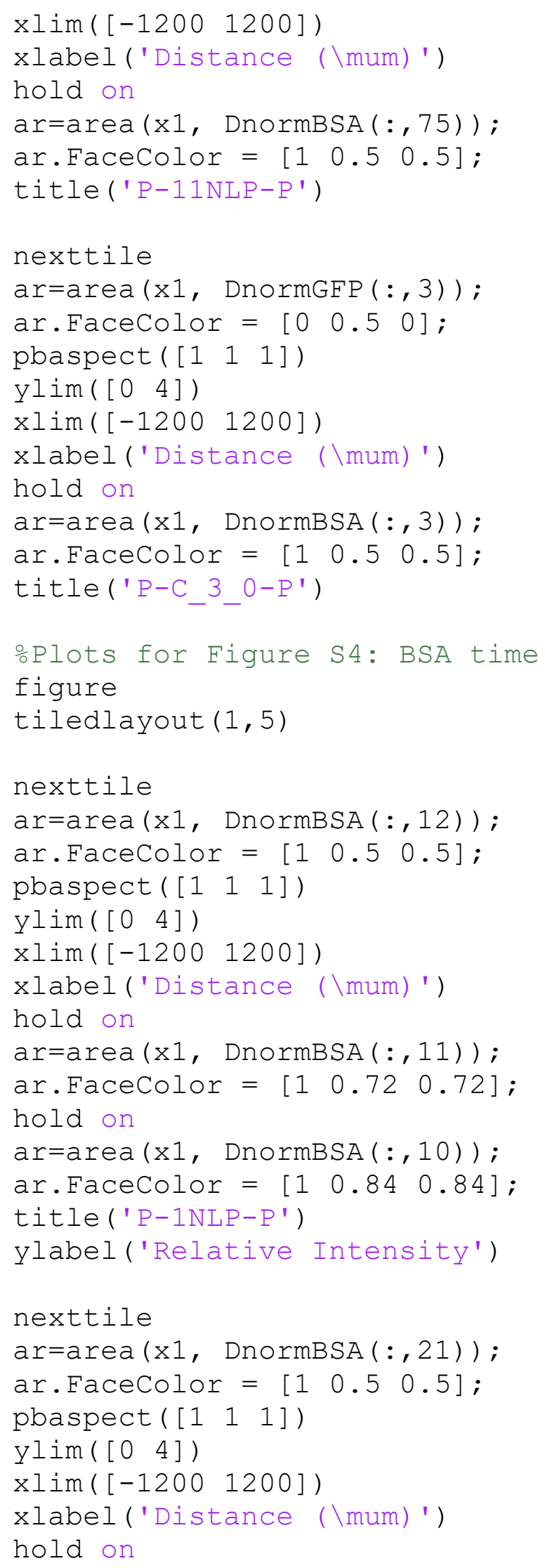




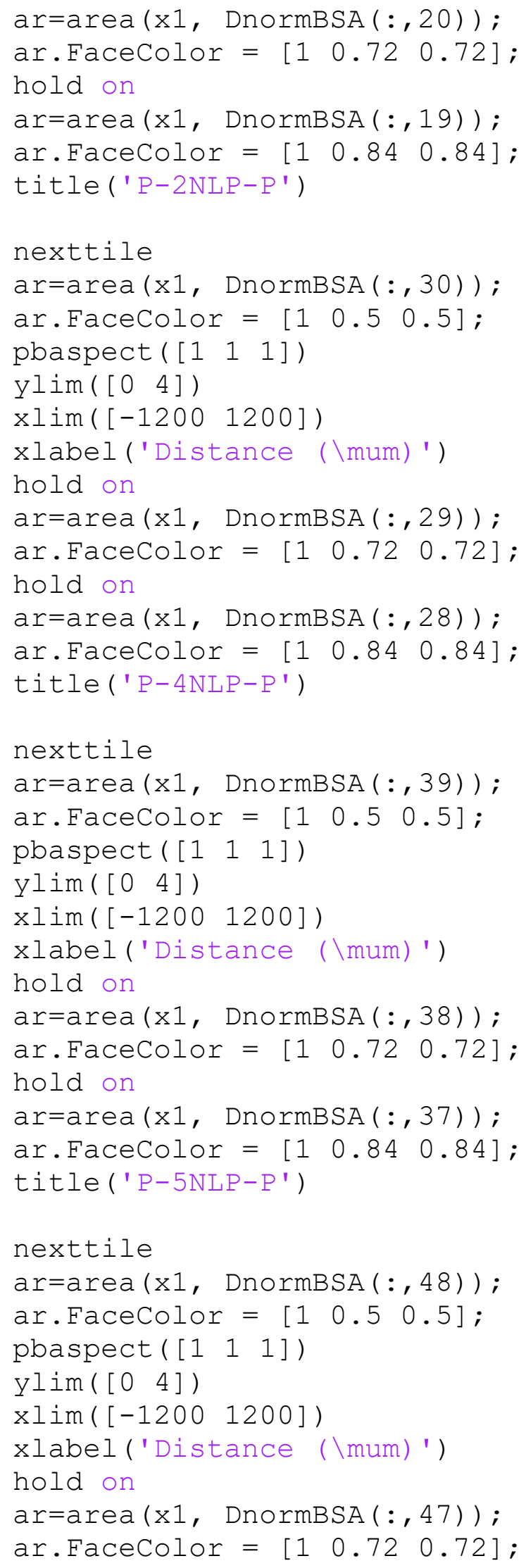




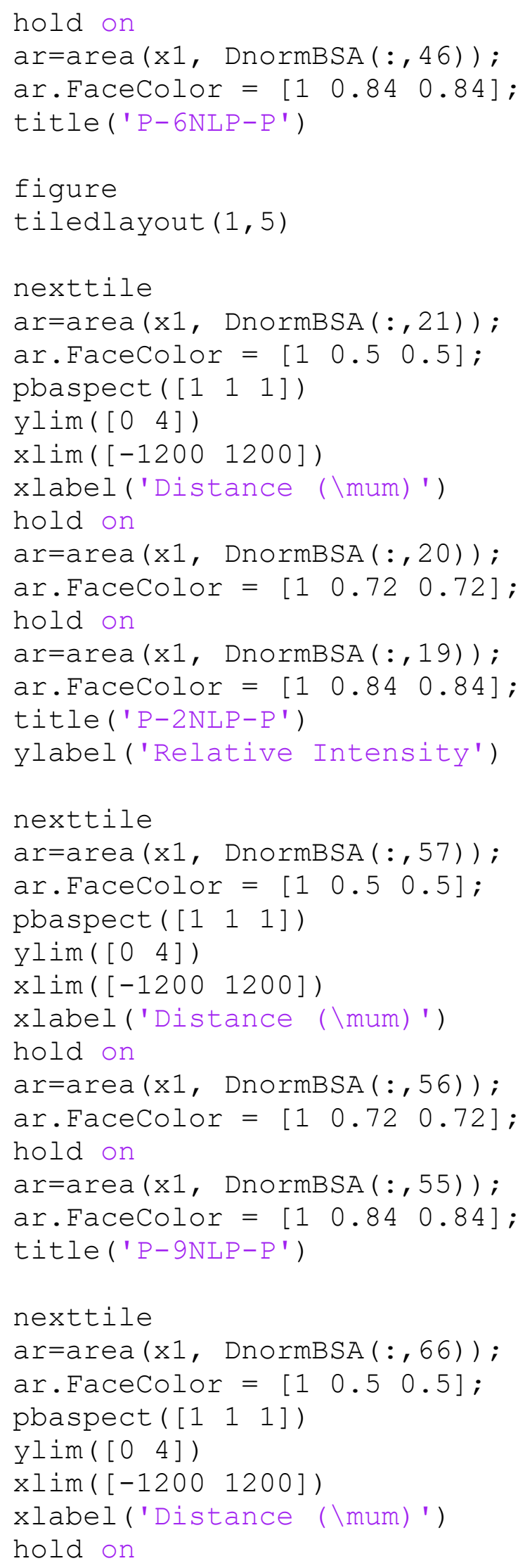




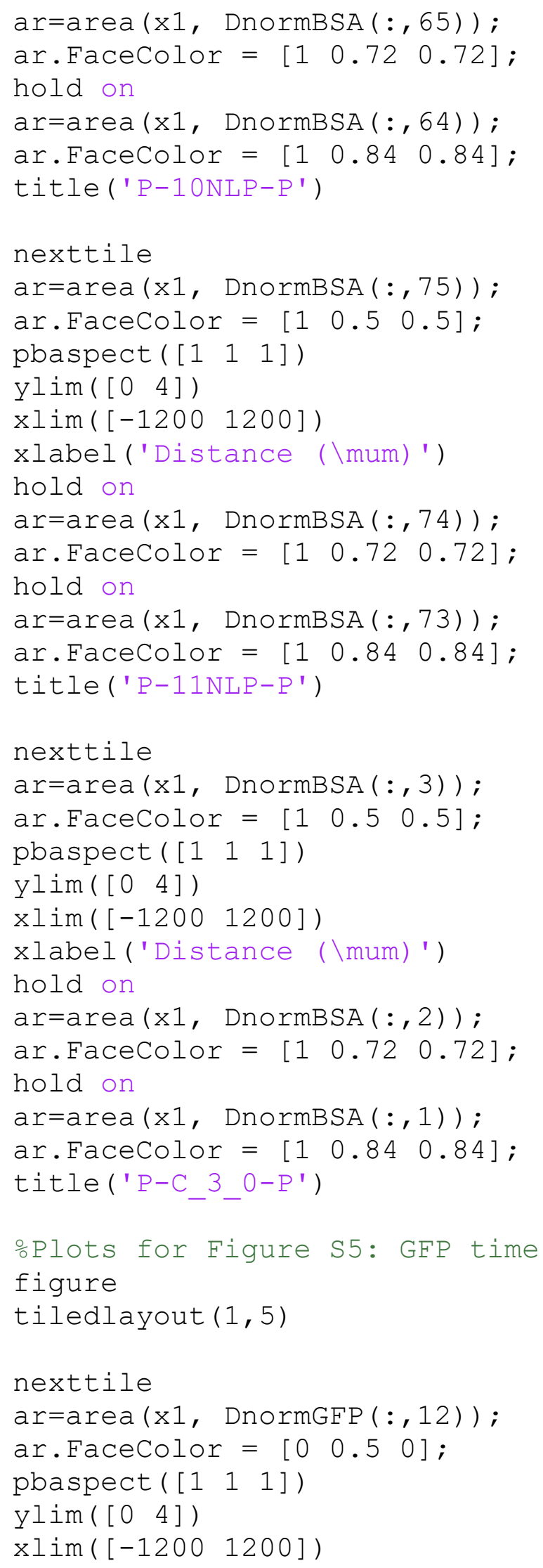




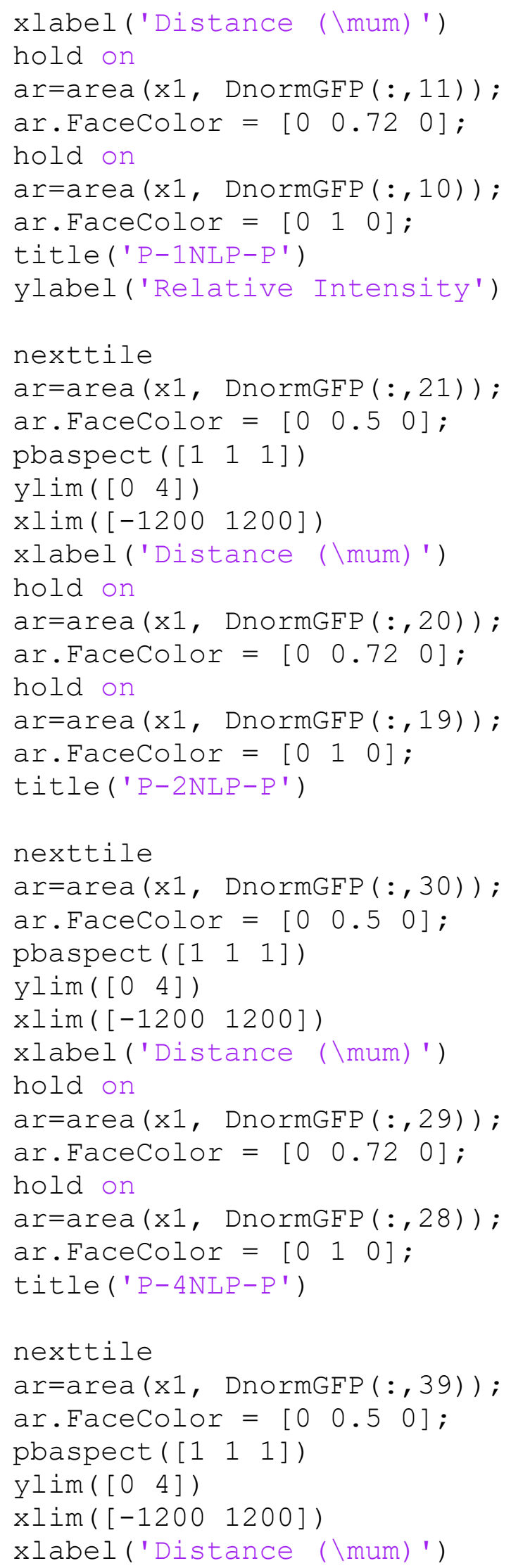




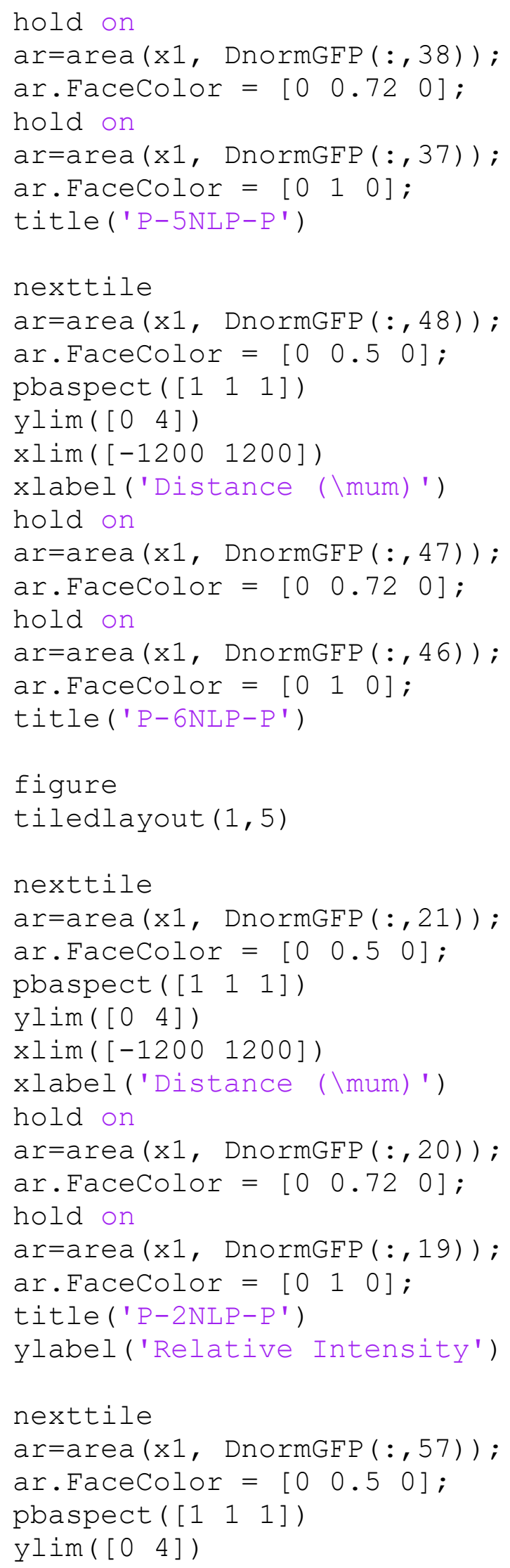




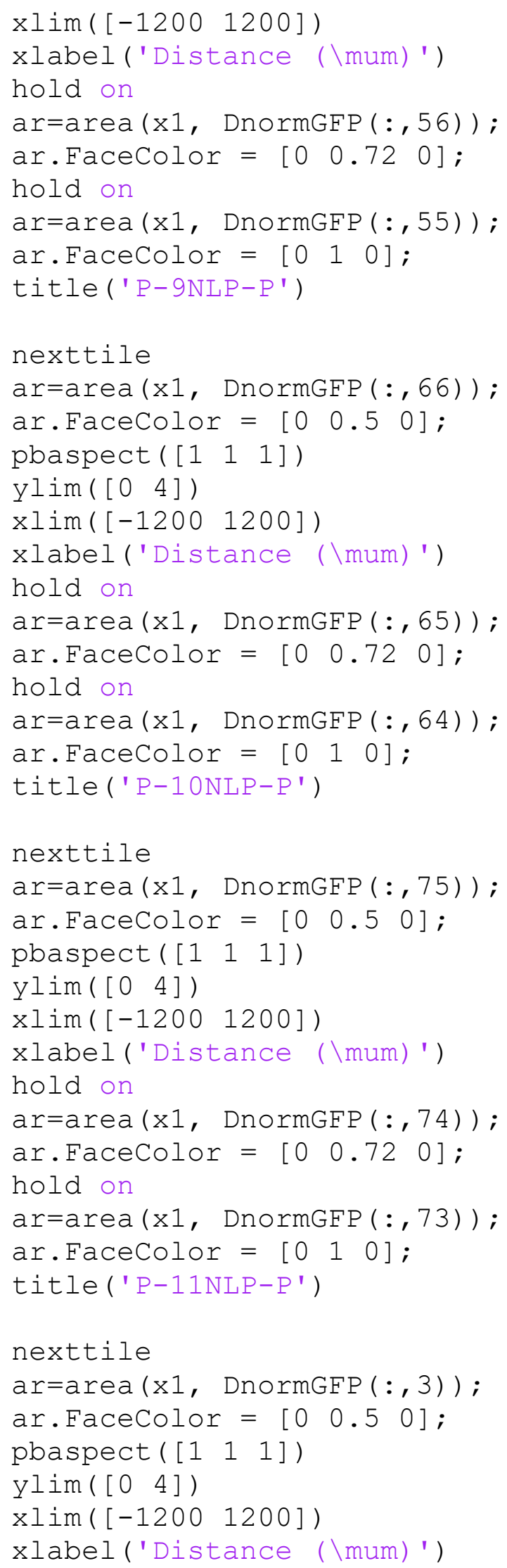




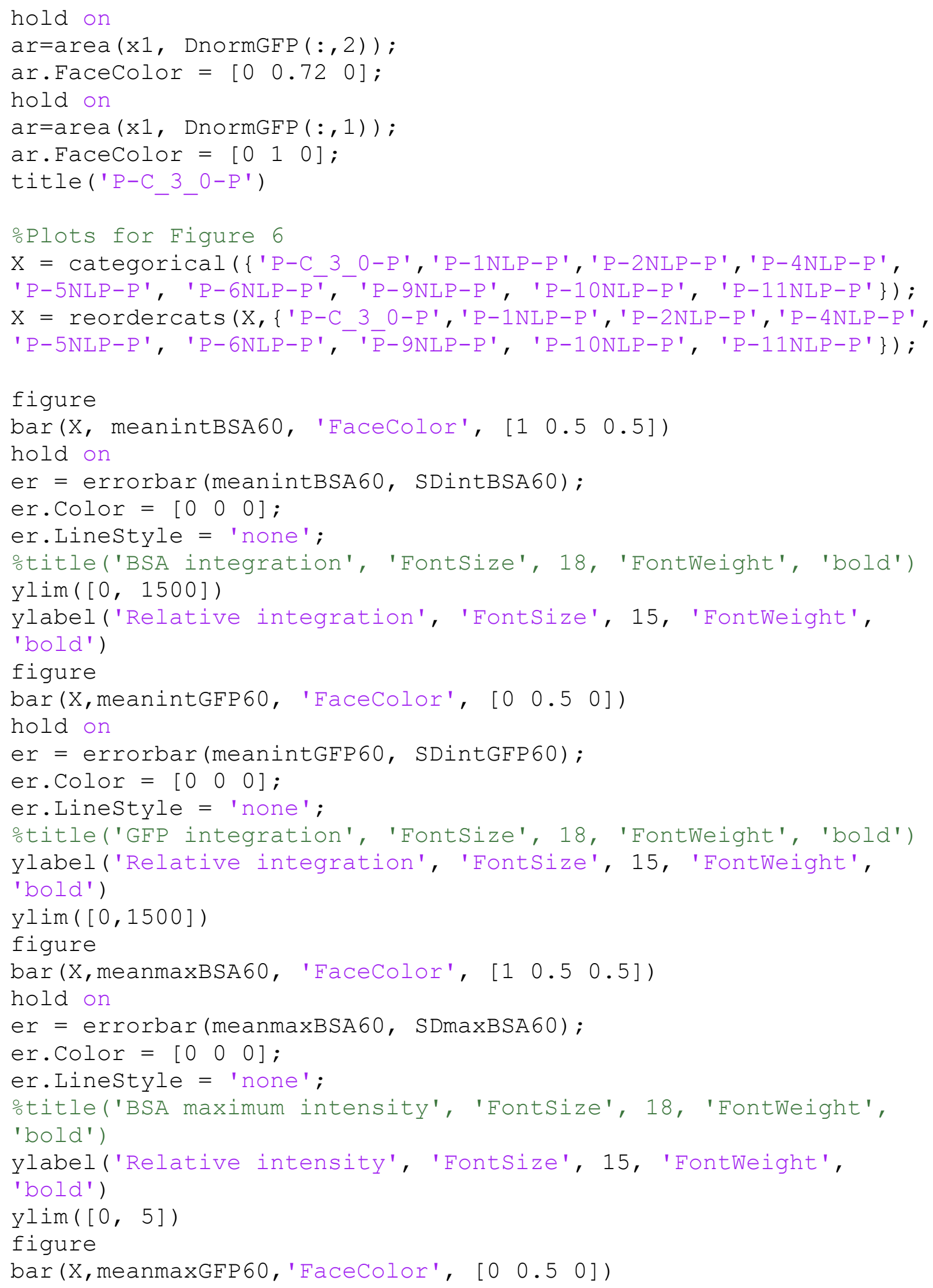




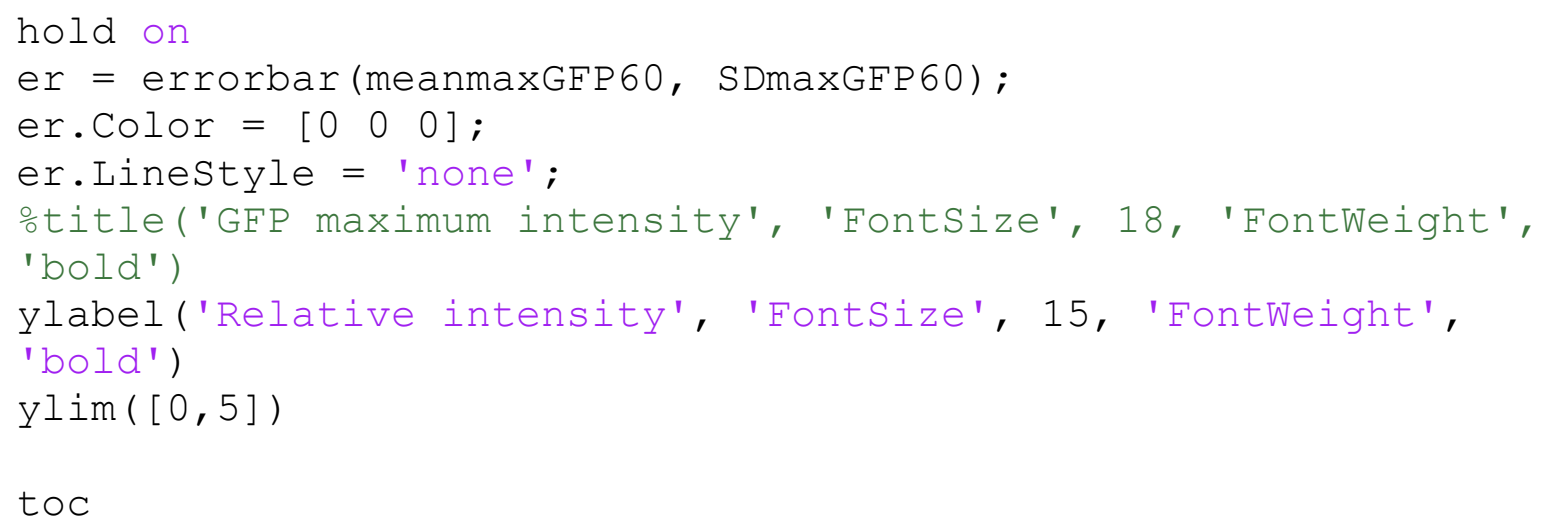

